\title{
Eight-and-a-half syndrome: one-and-a-half syndrome with peripheral facial nerve palsy
}

\author{
Ahmad Almutlaq MD, Alby Richard PhD MD \\ Cite as: CMAJ 2018 April 23;190:E510. doi: 10.1503/cmaj.180023
}

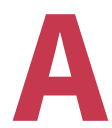

66-year-old man with right-hand dominance and a known history of hypertension and poorly controlled type 2 diabetes mellitus presented with a one-day history of abrupt-onset diplopia and right facial weakness. Extraocular examination showed left eye exotropia, impaired right eye adduction, conjugate rightward gaze palsy and gaze-evoked nystagmus when the patient looked to the left side (Appendix 1, video available at www.cmaj. ca/lookup/suppl/doi:10.1503/cmaj.180023/-/ DC1). These deficits persisted during pursuit and saccadic movements, but convergence and vertical eye movements were spared. The remainder of the physical examination was notable for right lower motor neuron facial weakness. Magnetic resonance imaging of the patient's brain obtained 24 hours after symptom onset showed a small focus of restricted diffusion in the right pontine tegmentum (Figure 1A). The patient's symptoms significantly improved with minimal residual sequelae.

One-and-a-half syndrome is characterized by conjugate horizontal gaze palsy in one direction in addition to contraversive internuclear ophthalmoplegia. ${ }^{1}$ The syndrome is usually caused by a unilateral lesion of the paramedian pontine reticular formation or the abducens nucleus, leading to conjugate gaze palsy toward the side of the lesion. There is also interruption of internuclear fibres of the medial longitudinal fasciculus after it has crossed the midline from its site of origin in the contralateral abducens nucleus, causing ipsilateral eye adduction deficit. The most common causes of one-anda-half syndrome include ischemic strokes, demyelinating lesions, tumours and arteriovenous malformations. ${ }^{2}$ The genu of the facial nerve loops around the abducens nucleus in the pontine tegmentum (Figure 1B), and lesions involving these fibres can result in ipsilateral lower motor neuron facial weakness. The current case shows "eight-and-a-half syndrome," which refers to one-and-a-half syndrome accompanied by a fascicular facial nerve lesion. ${ }^{3}$ Recognizing this specific constellation of findings allows for precise neuroanatomical localization to the right pontine tegmentum (Figure 1B), a region most frequently supplied by the anterior inferior cerebellar artery or paramedian pontine perforators of the basilar artery.

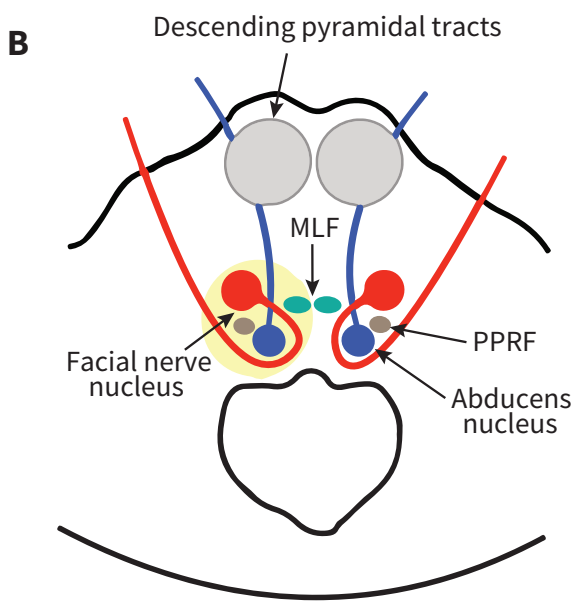

Figure 1: (A) Magnetic resonance imaging scan of the brain of a 66-year-old man, $24 \mathrm{~h}$ from symptom onset, with a $4 \times 8 \mathrm{~mm}$ area of restricted diffusion (arrow) in the right pontine tegmentum just anterior to the fourth ventricle on diffusion-weighted imaging sequence with corresponding low value on the apparent diffusion through the caudal pons, showing the genu of the facial nerve (red) looping around the abducens nucleus (blue) in the pontine tegmentum and location of the lesion (yellow) with relevant structures affected. Note: $\mathrm{MLF}=$ medial longitudinal fasciculus, $\mathrm{PPRF}=$ paramedian pontine reticular formation.

\section{References}

1. Fisher CM. Some neuro-opthalmological observations. J Neurol Neurosurg Psychiatry 1967;30:383-92.

2. Espinosa PS. Teaching Neurolmage: one-and-a-half syndrome. Neurology 2008;70:e20.

3. Eggenberger E. Eight-and-a-half syndrome: one-and-a half syndrome plus cranial nerve VII palsy. J Neuroophthalmol 1998;18:114-6.

\section{Competing interests: None declared.}

This article has been peer reviewed.

The authors have obtained patient consent.

Affiliations: Department of Neurology and Neurosurgery (Almutlaq, Richard), Montreal Neurological Institute, McGill University, Montréal, Que.; National Neuroscience Institute (Almutlaq), King Fahad Medical City, Riyadh, Saudi Arabia

Correspondence to: Alby Richard, alby.richard@gmail.com

Please see the following video online: Extraocular movement abnormalities in a 66-year-old man

www.cmaj.ca/lookup/suppl/doi:10.1503/cmaj.180023/-/DC1 